\title{
L'endoscopie, les risques du métier ?
}

\section{Heresbach}

(C) Springer-Verlag 2009

Cher(e)s Ami(e)s,

Après un premier numéro du journal «Acta Endoscopica » dans sa nouvelle formule, l'objectif doit être maintenant de trouver un rythme qui à la fois satisfasse le plus grand nombre tout en gardant une certaine rigueur éditoriale.

Quelques détails pratiques devront être améliorés comme la disponibilité du numéro lors des congrès de la SFED ou sous égide de la SFED et intégrer quelques diapositives, en particulier celles des algorithmes décisionnels ou de conclusion au texte concernant les communications lors de ces congrès tout en évitant l'écueil d'une revue qui ne serait qu'une succession de diapositives sans texte didactique ou explicatif.

Ce numéro aborde les risques de notre spécialité ou du métier d'endoscopiste, en particulier les deux qui sont le plus souvent cités et qui ont été débattus aux $11^{\text {es }}$ Journées de Réflexion de la SFED à Lyon le 24 janvier 2009 : le risque juridique et le risque infectieux. Si ces deux risques doivent être pris en compte, la publication de textes alarmants (entre 2 crises ou 2 guerres ou 2 parachutes dorés !) ne doit pas détourner les plus jeunes de notre spécialité et nous conforter dans notre pratique, qui pour la globalité, peut être qualifiée de bonne pratique.

L'endoscopie associée à un risque faible ou exceptionnel $(1 \%$ ou $1 \%$ ) trouve sa meilleure preuve dans la version 3 -dite d'accréditation des établissements - l'accréditement des hépato-gastroentérologues ne donnera pas

\footnotetext{
D. Heresbach $(\triangle)$
}

Rédacteur en chef

Service des Maladies de l'Appareil Digestif

CHU Pontchaillou, F-35033 Rennes Cedex 9, France

E-mail : denis.heresbach@chu-rennes.fr lieu à une réduction de leur cotisation de responsabilité civile professionnelle. Sans rentrer dans les détails, cela démontre bien que nous sommes une spécialité à risque « financièrement » négligeable ou négligé ! Sans rentrer dans le débat des différents types d'actes couverts par les différents niveaux de cotisation des responsabilités civiles professionnelles, ce sujet doit rester présent à nos esprits aussi bien pour l'activité des gastroentérologues libéraux et hospitaliers, ne serait-ce que par la nature et la quantité des moyens mis à disposition pour exercer nos actes en pleine sécurité, dont nous connaissons tous la maîtrise que nous en avons... à l'heure du travail en «flux tendu » et du principe de polyvalence.

L'endoscopie demeurera-t-elle un métier à risque car sa place est peu lisible dans le rapport et les recommandations pour le plan «Cancer 2009-2013». Vous pourrez constater que l'endoscopie n'est à aucun moment citée même si sa place comme moyen de dépistage de première ligne est discutable en l'absence de facteur de risque ; cependant, le maintien de plateaux techniques accessibles et de haute qualité demeure le garant d'une efficacité et minimisation des risques. Ainsi, dans la prise en charge multidisciplinaire des cancers avérés ou l'avis de l'oncologue polyvalent ou spécialiste d'organe est requis, celui de l'endoscopiste mériterait d'être considéré dans la séquence thérapeutique palliative ou adjuvante pour également minimiser les risques. Nous payons peut-être là les errements et les égarements des divergences de vue ou d'intérêt qui dans une certaine mesure peuvent nuire à la lisibilité de notre message.

Une fois de plus, ces dossiers ne pourront aboutir que si les différentes structures encadrant notre spécialité agissent conjointement et respectivement dans leur domaine de compétence : un état des lieux scientifique associé à des propositions organisationnelles par les Sociétés Savantes, 
un relais de ces propositions consensuelles auprès d'organisations professionnelles et une diffusion auprès des interlocuteurs politiques par les organismes syndicaux de notre profession. Seule, cette trilogie permettra d'atteindre l'objectif.
Veillons donc à regrouper nos forces pour que les 2 millions d'actes endoscopiques réalisés annuellement ne soient pas à la une des médias uniquement lorsqu'on parle de risque infectieux, de tarification, ou de complications, qui sans les nier, demeurent généralement exceptionnelles.

\section{Cours Méditerranéen d'Fndoscopie Digestive Thérapeutique}

\section{Monaco, 25-26 juin 2009}

Sous le patronage du Collège de Formation Continue de la Principauté de Monaco

\section{Organisation scientifique}

- Dr Rémi Dumas, Dr Jean-François Demarquay, Dr Patrick Hastier

\section{Ateliers interactifs}

Traitement endoscopique palliatif des sténoses biliaires

Extraction des prothèses plastiques et métalliques

Calculs difficiles : lithotritie extra ou intraductale

Coloscopie théropeutique : polypectomie, mucosectomie, dissection sous-muqueuse

Gestion endoscopique des complications de la polypectomie

\section{Démonstrations pratiques}

Traitement endoscopique du Barrett : mucosectomie, radiofréquence

Coloscopie thérapeutique : polypectomies, mucosectomies

Dissection sous-muqueuse

Microscopie confocale

Dilatations, prothèses du tractus digestif : œsophage, duodénum, côlon

Endoscopies biliopancréatiques thérapeutiques

Prothèses biliaires et pancréatiques

Cholangiopancréatoscopie diagnostique et thérapeutique

Lithotritie

Echoendoscopie interventionnelle

\section{Informations, inscriptions}

Centre Hospitalier Princesse Grace - Service d'Hépato-gastro-entérologie

Avenue Pasteur, F-98012 Monaco cedex - Tél. : (00 377) 97989805 - Fax : (00 377) 97989766

E-mail : stephanie.maestri@chpg.mc 